Gut, 1978, 19, 146-150

\title{
Variability of colonic function in healthy subjects
}

\author{
J. B. WYMAN, ${ }^{1}$ K. W. HEATON, A. P. MANNING, AND A. C. B. WICKS \\ From the University Department of Medicine, Bristol Royal Infirmary, Bristol
}

SUMMARY Twenty healthy subjects eating normal diets made repeated five-day stool collections, the 10 females making their collections in four to six successive weeks. In most subjects there were striking variations in transit time, measured by Hinton's method. The variability of average faecal wet and dry weight, faecal volume, and the frequency of defaecation was equally great, suggesting that the transit time variations were genuine. The size of individual stools varied even more, often tenfold or more. Faecal water content was relatively constant. There were no significant differences between males and females, and in the females there were no obvious changes related to the phases of the menstrual cycle. The normal variability of colonic function should be taken into account in planning experiments and in interpreting existing data.

In recent years interest has been renewed in the bulk and consistency of the faeces and in the intestinal transit time of healthy subjects, because correlations have been pointed out between these characteristics and the prevalence of various diseases of the large bowel, including appendicitis, diverticular disease, polyps, and carcinoma (Burkitt, 1971; Painter and Burkitt, 1971; Burkitt et al., 1972; Walker et al., 1973; Cleave, 1974). With so much emphasis being laid on these measurements, it is obviously important to know how much they vary from time to time in normal subjects. Surprisingly, little has been published on this point. When Hinton et al. (1969) described the technique of measuring whole-gut transit time with the use of radio-opaque plastic pellets, they reported that, in 25 healthy men, duplicate measurements varied by as much as three days. This variability has sometimes been taken as indicating poor repeatability of the method (Eastwood et al., 1974; Cummings et al., 1976). Certainly, some variability is inevitable from the fact that the method uses as an arbitrary end-point the time at which the stool is passed containing the 20th of 25 ingested pellets. However, it is possible that much of the variability is due to genuine biological variation. Common experience suggests that colonic function does vary considerably from time to time, especially in those who travel or lead irregular

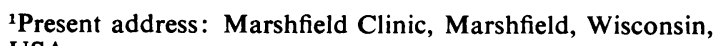
USA.

Received for publication 2 September 1977 or stressful lives. If biological variation is the main explanation of variable transit measurements, then other parameters of colonic function should vary in time to a similar degree. We have examined the variability of faecal weight, volume, and frequency, as well as of transit time measured by Hinton's method, in 20 healthy subjects. Since these included 10 women, we took the opportunity of looking for rhythmical variations of colonic function during the normal menstrual cycle.

\section{Methods}

\section{SUBJECTS}

The subjects studied were 10 males and 10 females, aged 24 to 43 years except for one boy aged 11 years and one girl aged 15 years, all pursuing their normal activities. The women were asked to eat their usual diet but to attempt to eat about the same amount of fibre-containing foods every day. The only drugs taken were contraceptive pills by four and an iron preparation and an antihypertensive by one. The men used no medications and were given written dietary instructions aimed at standardising their daily intake of fibre.

\section{PROCEDURE}

Each subject was provided with a convenient odourfree stool collection kit, contained in an unobtrusive case which could be taken to the place of work (Wyman et al., 1977, in preparation). This method was similar to the systems described by Hoffman 
et al. (1973) and Glober et al. (1974). Stool collections always began on a Sunday morning and continued for five days. The women began their first collection on the Sunday after menstrual flow had begun and made further collections each week till the menses recurred. The men each made two collections, separated by three to 11 weeks (Wyman et al., 1976).

Transit time was determined by the method of Hinton et al. (1969), using $253 \mathrm{~mm}$ bariumimpregnated polyethylene pellets (Portex Ltd., Hythe, Kent), and the time of appearance of $80 \%$ of the pellets as the end-point. Stools were weighed and $x$-rayed individually, then each five-day collection was pooled and blended with a roughly equal, known volume of water. Subtraction of the water volume yielded an estimate of faecal volume. Faecal dry weight was calculated from the weight of a freeze-dried aliquot of known volume. Defaecation frequency and the average interval between bowel actions was calculated from the recorded dates and times of individual stools.

\section{Results}

In all, 69 five-day stool collections from the 20 subjects were available for analysis. Table 1 sum- marises the individual results in the 10 females for each of the eight parameters studied. Each female made between four and six collections. This permitted the calculation of coefficients of variation for each subject for each measurement. These could then be compared statistically to determine whether transit time was a more variable measurement than the other parameters. Table 2 shows that there is no significant difference between the coefficients of variation for transit time and those for faecal wet weight, dry weight, volume, frequency, or interval. Individual stool size was significantly more variable, and water content was significantly less variable than any of the other measurements.

Table 3 shows the results of the duplicate studies in the 10 males. In this case, the small number of studies does not permit statistical analysis, but the variability of the transit time data is not obviously greater than that of the other measurements.

Figure 1 displays the results of all 69 transit time measurements. In nine tests (all in females), $80 \%$ of the radio-opaque pellets failed to pass during the five-day period of the stool collection. These prolonged transit times, which occurred in five women (three times in one of them) are recorded as the time

Table 1 Intestinal transit time and faecal measurement of 10 females

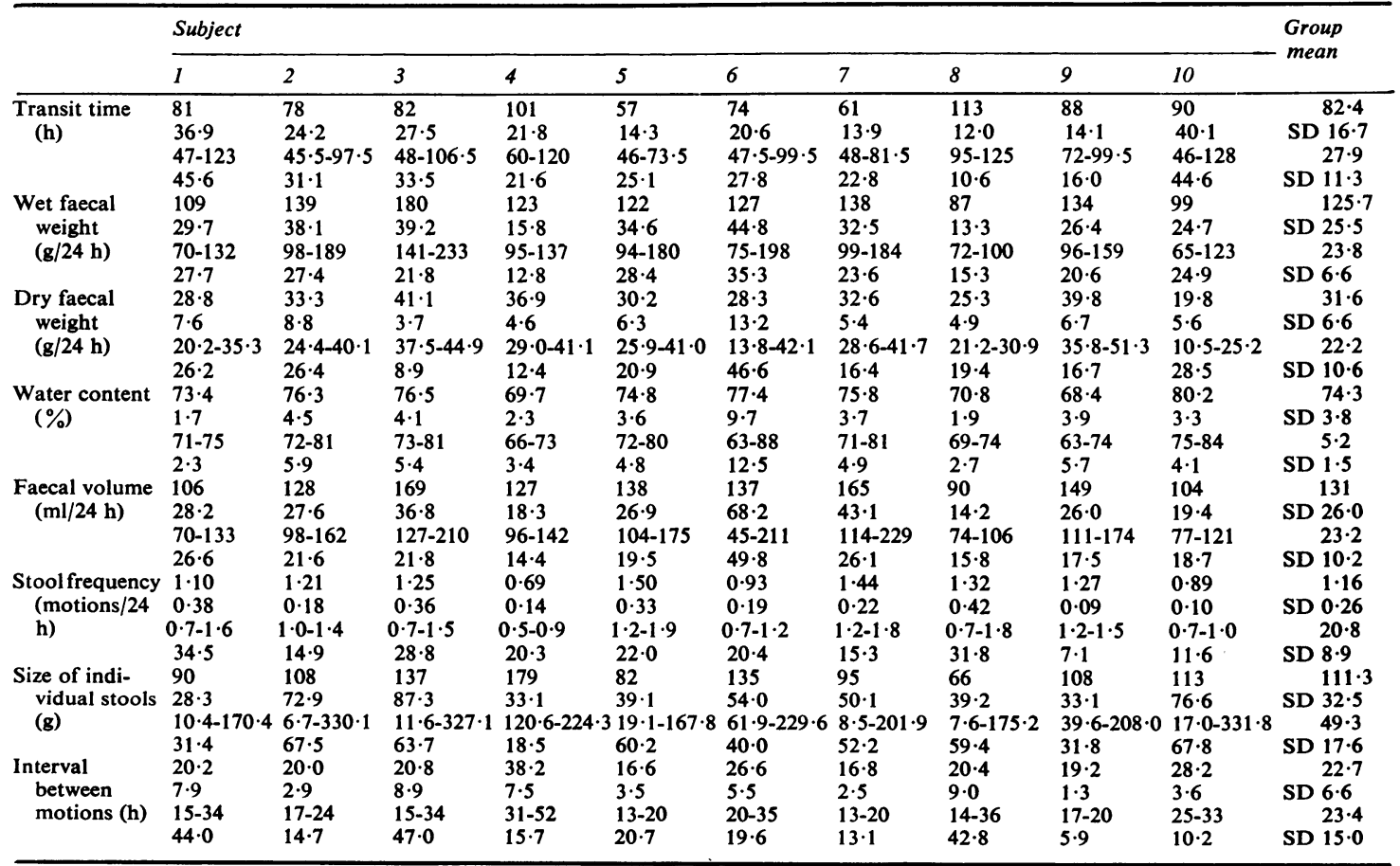

In each block are shown mean, standard deviation, range, and coefficient of variation. In the group mean column are shown the means of the means and coefficients of variation, with standard deviations. Forty-nine five-day collections were made, with each subject making four to six collections. 
Table 2 Coefficients of variation for transit time and faecal measurements in 10 healthy women

\begin{tabular}{lrrrr}
\hline & & \multicolumn{3}{c}{$\begin{array}{c}\text { Comparison with } \\
\text { transit time }\end{array}$} \\
\cline { 3 - 5 } & Mean & $S D$ & $t$ & \multicolumn{2}{c}{$\mathrm{P}$} \\
\hline Transit time & 27.9 & 11.3 & & - \\
Wet faecal weight & 23.8 & 6.6 & 1.31 & $\mathrm{NS}$ \\
Dry faecal weight & 22.2 & 10.6 & 1.37 & $\mathrm{NS}$ \\
Water content & 5.2 & 1.5 & 6.09 & $<0.001$ \\
Faecal volume & 23.2 & 10.2 & 1.10 & NS \\
Stool frequency & 20.8 & 8.9 & 1.69 & NS \\
Individual stool size & 49.3 & 17.6 & -3.54 & $<0.001$ \\
Interval between & & & & \\
motions & 23.4 & 15.0 & 0.81 & NS \\
\hline
\end{tabular}

Each faecal measurement is compared to transit time using a paired $t$ test.

of the total faecal collection. Figure 2 displays the results of 69 measurements of daily faecal wet weight. Figure 3 shows the results of 68 measurements of daily faecal dry weight. One aliquot (from subject 2) was lost during freeze drying. The figures show the great variability of colonic function both within and between individuals.

Using both Student's $t$ and rank tests there was no significant difference between men and women in any of these measurements. No relationship was seen to age, body weight, or body surface area. Only in females was there a significant negative correlation between transit time and wet faecal weight $(r=0.633$; $\mathrm{P}<0.05)$. The males demonstrated a significant correlation between individual stool size and dry weight $(r=0.793 ; \mathrm{P}<0.01)$.

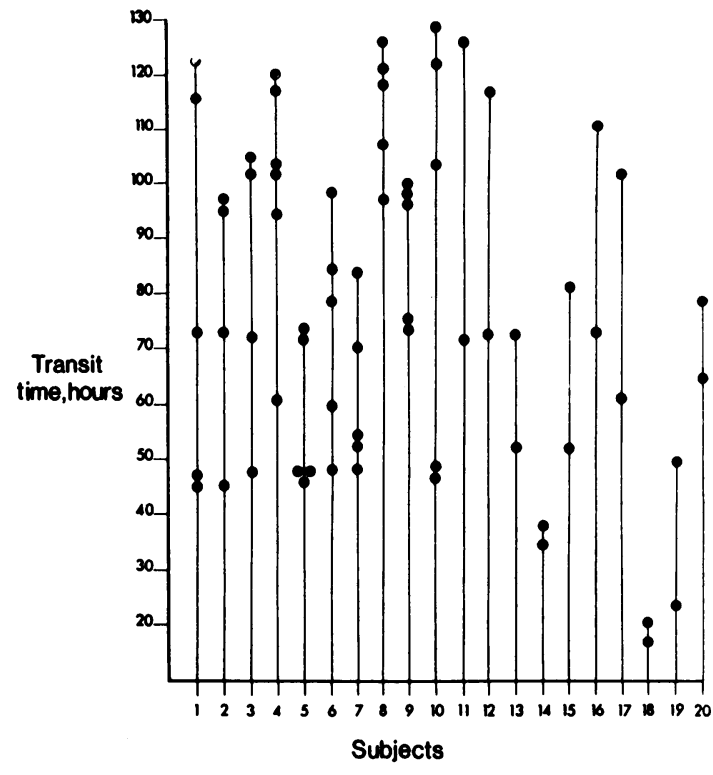

Fig. 1 Intestinal transit times in hours. Results of 69 five-day faecal collections made by 20 subjects. Each subject made two to six collections. Each closed circle represents an individual collection by subjects 1-20 whose results are summarised in Tables 1 and 3. The double circles represent the results in five subjects making nine collections who failed to pass $80 \%$ of the ingested pellets; total collection time is considered transit time in these subjects.

Table 3 Intestinal transit time and faecal measurements of 10 males

\begin{tabular}{|c|c|c|c|c|c|c|c|c|c|c|c|}
\hline & \multicolumn{10}{|l|}{ Subject } & \multirow{2}{*}{$\begin{array}{l}\text { Group } \\
\text { mean }\end{array}$} \\
\hline & 11 & 12 & 13 & 14 & 15 & 16 & 17 & 18 & 19 & 20 & \\
\hline $\begin{array}{l}\text { Water content } \\
(\%) \\
\text { Faecal volume } \\
\text { (ml/24 h) } \\
\text { Stool fre- } \\
\text { quency } \\
\text { (motions } / 24 \mathrm{~h} \text { ) }\end{array}$ & $\begin{array}{l}71 \\
70,72 \\
82 \\
69,95 \\
1 \cdot 8 \\
0 \cdot 6-2 \cdot 9 \\
0\end{array}$ & $\begin{array}{l}70 \\
66,74 \\
181 \\
139,222 \\
2 \cdot 3 \\
1 \cdot 9-2 \cdot 9\end{array}$ & $\begin{array}{l}74 \\
73,75 \\
185 \\
175,195 \\
1 \cdot 0 \\
0 \cdot 5 \cdot 1 \cdot 7\end{array}$ & $\begin{array}{l}76 \\
70,81 \\
98 \\
92,103 \\
2 \cdot 0 \\
1 \cdot 9-2 \cdot 1\end{array}$ & $\begin{array}{l}76 \\
69,83 \\
150 \\
147,153 \\
1 \cdot 3 \\
0 \cdot 9-2 \cdot 0\end{array}$ & $\begin{array}{l}71 \\
70,71 \\
85 \\
65,104 \\
1 \cdot 1 \\
0 \cdot 3-2 \cdot 0\end{array}$ & $\begin{array}{l}76 \\
74,77 \\
150 \\
120,180 \\
1 \cdot 2 \\
0 \cdot 3-2 \cdot 8\end{array}$ & $\begin{array}{l}83 \\
82,83 \\
196 \\
193,198 \\
1 \cdot 0 \\
0 \cdot 9-1 \cdot 0\end{array}$ & $\begin{array}{l}73 \\
70,76 \\
130 \\
122,138 \\
1 \cdot 0 \\
0 \cdot 9-1 \cdot 1\end{array}$ & $\begin{array}{l}82 \\
80,84 \\
127 \\
120,134 \\
0 \cdot 7 \\
0 \cdot 1-1 \cdot 0\end{array}$ & $\begin{array}{c}75 \cdot 0 \\
\text { SD } 4 \cdot 3 \\
138 \cdot 2 \\
\text { SD } 41 \cdot 4 \\
0.96 \\
\text { SD } 0 \cdot 32\end{array}$ \\
\hline
\end{tabular}

In each block are shown the mean and two individual values. In addition, standard deviation is shown for size of individual stools, interval between bowel motions, and group mean. Each subject made two five-day faecal collections. 


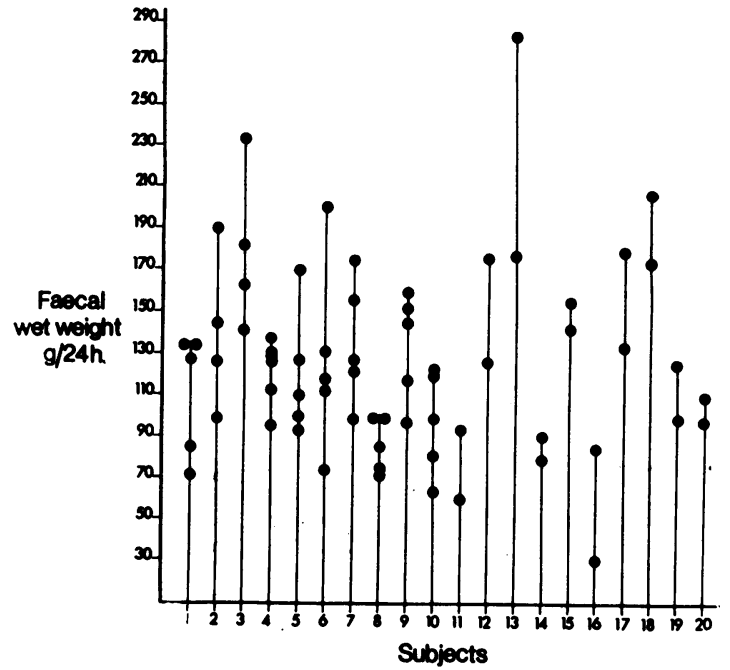

Fig. 2 Faecal wet weight in g/24 hours. Results of 69 five-day faecal collections made by 20 subjects. Each subject made two to six collections. Each closed circle represents in individual collection by subjects 1-20 whose results are summarised in Tables 1 and 3 . The double circles represent the results in five subjects who failed to pass $80 \%$ of the ingested pellets.

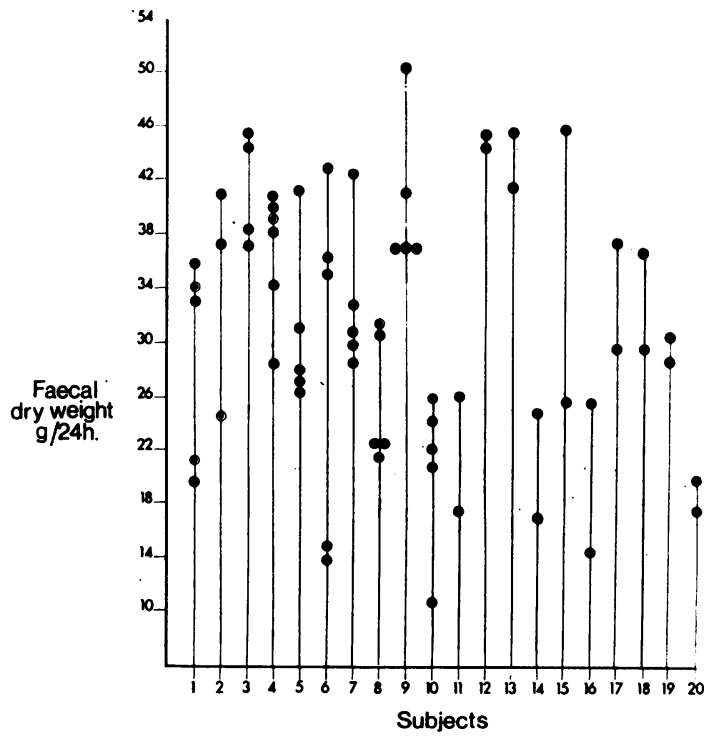

Fig. 3 Faecal dry weight in g/24 hours. Results of 68 five-day faecal collections made by 20 subjects. Each subject made two to six collections. Each closed circle represents an individual collection by subjects 1-20 whose results are summarised in Tables 1 and 3. The double circles represent the results of five subjects who failed to pass $80 \%$ of the ingested pellets.
Individual stool size was significantly correlated with the interval between bowel motions in women $(r=0.806 ; \mathrm{P}<0.01)$, but not in the males. There was no correlation in either sex of transit time and dry weight, transit time and individual stool size, interval between bowel motions and wet faecal weight or dry faecal weight, or individual stool size and wet faecal weight.

In the females, there was no clear relationship between transit time or faecal measurements and the phases of the menstrual cycle. Endocrinologically, there are three main phases during a 28-day menstrual cycle - the oestrogen peak phase which is around ovulation, the progesterone peak phase which is around nine days later (and is associated with a second smaller oestrogen peak), and a lowoestrogen, low-progesterone phase which is roughly eight days in length and contains the menstrual flow. The design of our study was such that the stool collection periods were not necessarily included within the three endocrine phases. The six women not taking contraceptive tablets had menstrual cycles of 22 to 35 days. If their data are related to a theoretical 28-day cycle, 23 of their 30 stool collections (at least four of the five days) fell into one of the three menstrual endocrine phases. Of these 23 collections, eight were made during the low-oestrogen, low-progesterone phase, seven during the oestrogen peak phase, and eight during the progesterone peak phase. Statistical comparison of the data from the three phases showed no significant differences.

\section{Discussion}

This study has shown wide variations in faecal measurements and transit times, not only between individuals but also on repeated measurement within the same individual. These variations were as great in average faecal wet and dry weight and faecal volume, measured over five days, as in transit time measured over a similar period, and substantially greater in the case of individual stool size. This suggests that much if not most of the observed variability of transit time as measured by Hinton's method is due to genuine biological variations in colonic function.

In support of this belief is the fact that the more complicated measurement of 'mean transit time' does little to reduce person to person variation. Thus, Cummings et al. (1976) found the mean transit time to range from 0.7 to 4.0 days in their studies on six healthy subjects, and this variability persisted when the subjects ate a standard diet, the range still being 1.2 to 4.8 days. Nevertheless, for accurate comparisons of transit time in different groups and 
on different regimes it is probably desirable to use a mean transit time technique, because this eliminates the arbitrariness of a particular cut-off point such as the $80 \%$ passage of pellets. The single stool technique of Cummings and Wiggins (1976) appears attractively simple as well as accurate.

The inherent variability of faecal and transit measurements probably explains many apparent inconsistencies in the reported effects of dietary fibre on colonic function (Fantus et al., 1941; Hoppert and Clark, 1942; Streicher and Quirk, 1943; Eastwood et al., 1973; Connell and Smith, 1974; Findlay et al., 1974; Parks, 1974; Durrington et al., 1976). When a subject is given bran, he may, by chance, be about to experience a large prolongation of transit time. In this event, although bran may result in the prolongation being less than it would have been, nevertheless, to an observer it will seem as if bran has been responsible for prolonging his transit time. Experience with the mean transit time technique, though limited, suggests that ironing-out day to day variations in transit rate does make the effects of bran appear more consistent (Cummings et al., 1976).

The extraordinary variation in the size of individual stools indicates the great importance of collecting stools for several days when meaningful information is required about an individual's stool weight. In contrast, our study showed remarkably little variation in the water content of the stools.

We are unable to explain why only in the women was there found to be a correlation between transit time and wet weight, and individual stool size and the interval between bowel motions. Similarly, we cannot explain why only in the males was there a correlation between individual stool size and faecal dry weight.

Our attempt to relate transit time and faecal measurements to the phases of the menstrual cycle was unsuccessful, but this could have been due to the small numbers involved as well as to the unknown variable of the time of ovulation. The assignment of faecal collections to the various endocrine phases was not supported by serum hormone measurements. It has been claimed that $64 \%$ of women notice a change in bowel habit with menstruation (Rees and Rhodes, 1976). We found no objective evidence of this and are not aware that any such evidence has been published.

In conclusion, it is clear that healthy subjects show wide spontaneous variations in colonic function. These must be taken into account in planning studies on the effects of drugs or diets on colonic behaviour and in making epidemiological comparisons.

\section{References}

Burkitt, D. P. (1971). Epidemiology of cancer of the colon and rectum. Cancer, 28, 3-13.

Burkitt, D. P., Walker, A. R. P., and Painter, N. S. (1972). Effect of dietary fibre on stools and transit-times, and its role in the causation of disease. Lancet, 2, 1408-1411.

Cleave, T. L. (1974). The Saccharine Disease. Wright: Bristol.

Connell, A. M., and Smith, C. L. (1974). The effect of dietary fibre on transit time. In Proceedings of the 4th International Symposium on Gastrointestinal Motility, pp. 365-368. Mitchell Press: Vancouver.

Cummings, J. H., and Wiggins, H. S. (1976). Transit through the gut measured by analysis of a single stool. Gut, 17, 219-223.

Cummings, J. H., Jenkins, D. J. A., and Wiggins, H. S. (1976). Measurement of the mean transit time of dietary residue through the human gut. Gut, 17, 210-218.

Durrington, P. N., Manning, A. P., Bolton, C. H., and Hartog, M. (1976). Effect of pectin on serum lipids and lipoproteins, whole-gut transit-time, and stool weight. Lancet, 2, 394-396.

Eastwood, M. A., Fisher, N., Greenwood, C. T., and Hutchinson, J. B. (1974). Perspectives on the bran hypothesis. Lancet, 1, 1029-1033.

Eastwood, M. A., Kirkpatrick, J. R., Mitchell, W. D., Bone, A., and Hamilton, T. (1973). Effects of dietary supplements of wheat bran and cellulose on faeces and bowel function. British Medical Journal, 4, 392-394.

Fantus, B., Hirschberg, N., and Frankl, W. (1941). The mode of action of bran. II. Influence of size and shape of bran particles and of crude fiber isolated from bran. Review of Gastroenterology, 8, 277-280.

Findlay, J. M., Smith, A. N., Mitchell, W. D., Anderson, A. J. B., and Eastwood, M. A. (1974). Effects of unprocessed bran on colon function in normal subjects and in diverticular disease. Lancet, 1, 146-149.

Glober, G. A., Klein, K. L., Moore, J. O., and Abba, B. C. (1974). Bowel transit-times in two populations experiencing similar colon-cancer risks. Lancet, 2, 80-81.

Hinton, J. M., Lennard-Jones, J. E., and Young, A. C. (1969). A new method for studying gut transit times using radioopaque markers. Gut, $10,842-847$.

Hoffman, N. E., La Russo, N. F., and Hoffman, A. F. (1973). An improved method for faecal collection: the faecal field kit. Lancet, 1, 1422-1423.

Hoppert, C. A., and Clark, A. J. (1942). Bran muffins and normal laxation. Journal of the American Dietetic Association, 18, 524-525.

Painter, N. S., and Burkitt, D. P. (1971). Diverticular disease of the colon: a deficiency disease of western civilization. British Medical Journal, 2, 450-454.

Parks, T. G. (1974). The effects of low and high residue diets on the role of transit and composition of the faeces. In Proceedings of the 4th International Symposium on Gastrointestinal Motility, pp. 369-379. Nitchell Press: Vancouve

Rees, W. D. W., and Rhodes, J. (1976). Altered bowel habit and menstruation. Lancet, $2,475$.

Streicher, M. H., and Quirk, L. (1943). Constipation: clinical and roentgenologic evaluation of the use of bran. American Journal of Digestive Diseases, 10, 179-181.

Walker, A. R. P., Richardson, B. D., and Walker, B. F. (1973). Appendicitis, fibre intake, and bowel behaviour in ethnic groups in South Africa. Postgraduate Medical Journal, 49, 243-249.

Wyman, J. B., Heaton, K. W., Manning, A. P., and Wicks, A. C. B. (1976). The effect on intestinial transit and the feces of raw and cooked bran in different doses. American Journal of Clinical Nutrition, 29, 1474-1479. 